\title{
Ion Trap Mass Spectrometry Affords Advances in the Analytical and Atmospheric Chemistry of 2-Hydroxy-2-Methylpropanal, a Proposed Photooxidation Product of 2-Methyl-3-Buten-2-O1
}

\author{
Reggie Spaulding and M. Judith Charles \\ Department of Environmental Toxicology, University of California, Davis, Davis, California, USA
}

Ernesto C. Tuazon

Air Pollution Research Center, University of California, Riverside, Riverside, California, USA

Matthew Lashley

Department of Chemistry, University of California, Davis, Davis, California, USA

In the western United States, in areas where emissions of the biogenic hydrocarbon, 2-methyl-3-buten-2-ol (MBO) are high, $\mathrm{MBO}$ contributes significantly to the oxidative capacity of the atmosphere. Hydroxyl radical oxidation of $\mathrm{MBO}$ can play an important role in forming tropospheric ozone, and $\mathrm{MBO}$ reaction products may contribute to the formation of secondary organic aerosols [1-3]. Although 2-hdyroxy-2-methylpropanal was tentatively identified as a product from the reaction of $\mathrm{MBO}$ with $\cdot \mathrm{OH}$ in indoor chamber studies, the identity of the compound was not confirmed due to the lack of an authentic standard. Further, no data exists on the atmospheric generation and fate of 2-hydroxy-2-methylpropanal in the ambient environment. Herein, we provide further evidence that 2-hydroxy-2-methylpropanal is generated by $\cdot \mathrm{OH}$ reaction with $\mathrm{MBO}$ by identifying 2-hydroxy-2-methylpropanal in an indoor chamber experiment and in ambient air sampled in the Blodgett Forest, where MBO emissions are high. We analyzed 2-hydroxy-2-methylpropanal by using a method that relies on $O-(2,3,4,5,6$-pentafluorobenzyl)hydroxylamine (PFBHA) and bis-(trimethylsilyl) trifluoroacetamide (BSTFA) derivatization along with ion-trap mass spectrometry. Tentative identification of 2-hydroxy-2-methylpropanal was possible by using knowledge gained in this study regarding the mass spectrometry of PFBHA-BSTFA derivatives of carbonyls with primary, secondary, and tertiary $-\mathrm{OH}$ groups, and ado- and keto-acids. The identification was confirmed by comparing the methane CI mass spectra and relative gas chromatographic retention time obtained by analyzing 2-hydroxy-2-methylpropanal in a sample extract and a synthesized authentic standard. Since the standard became available at the end of this study (after all samples were analyzed), we also developed a method for semi-quantification of 2-hydroxy-2-methylpropanal, with a detection limit of $27 \mathrm{pptv}$ in air. We used the method to provide the first ambient air measurements of 2-hydroxy-2-methylpropanal. The analyte is not commercially available, and hence other researchers who have not synthesized an authentic standard can employ the method. (J Am Soc Mass Spectrom 2002, 13, 530-542) (C) 2002 American Society for Mass Spectrometry

$\mathrm{B}$ iogenic volatile organic compounds (VOC) play an important role in tropospheric ozone and secondary organic aerosol formation in rural and urban areas, and can dominate the oxidative capacity of the atmosphere in rural areas [2-7]. On a global scale,

Published online April 2, 2002

Address reprint requests to Dr. M. J. Charles, Department of Environmental Toxicology, University of California, Davis, One Shields Avenue, Davis, California 95616-8588. E-mail: mjcharles@ucdavis.edu biogenic emissions exceed anthropogenic emissions by approximately 10 times [8]. Isoprene is the dominant biogenic hydrocarbon and constitutes about $30 \%$ of the biogenic VOCs in North America [8]. Although MBO comprises only $5 \%$ of the biogenic VOCs in North America, $\mathrm{MBO}$ concentrations were measured at 2 to 7 times the concentrations of isoprene in the Blodgett Forest, CA, and at Niwot Ridge, CO [2, 9], because of emission from ponderosa and lodgepole pines, trees that are endemic to western North America [10]. Hy- 


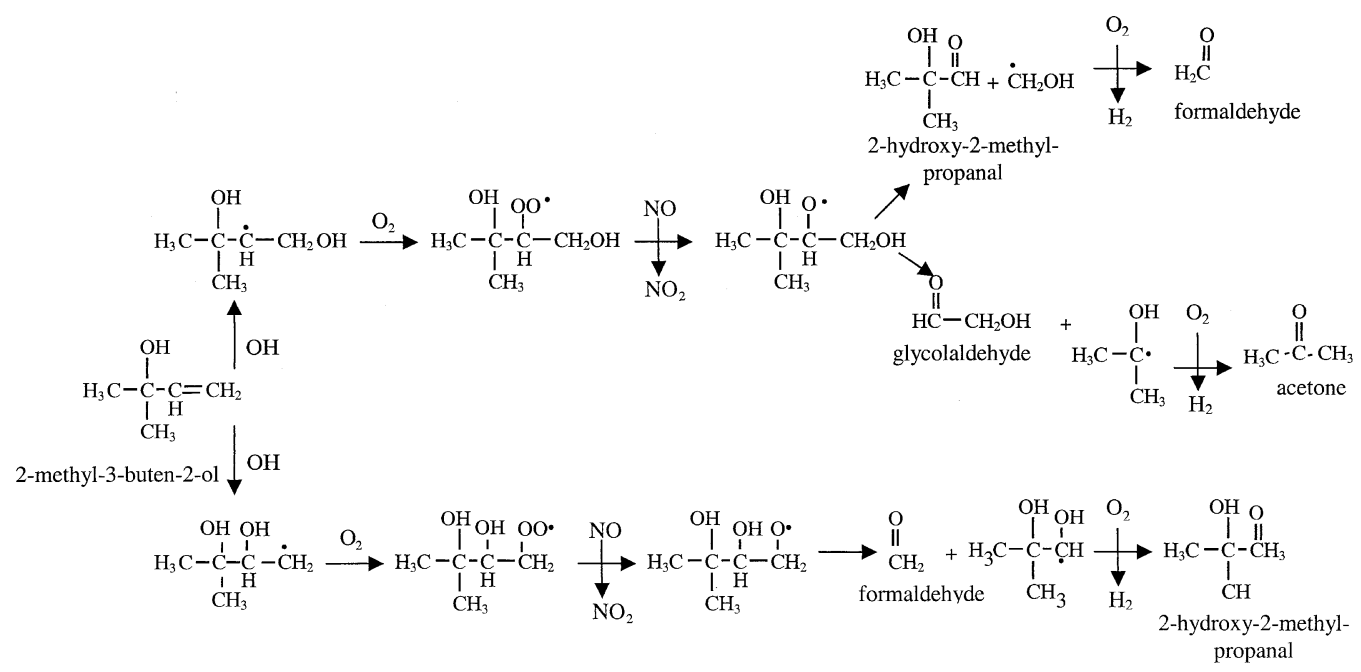

Figure 1. Mechanism for reaction of $\mathrm{MBO}$ with $\cdot \mathrm{OH}$ proposed by Fantechi et al. [13].

droxyl radical oxidation is the dominant loss mechanism for MBO during daytime [11, 12]. Therefore, in order to reduce tropospheric ozone in atmospheres that are affected by MBO emissions, it is essential that we gain insight into atmospheric processes affecting $\cdot \mathrm{OH}$ oxidation of $\mathrm{MBO}$, and the generation and fate of the reaction products.

Laboratory (chamber) studies establish that $\cdot \mathrm{OH}$ oxidation of $\mathrm{MBO}$ yields acetone and glycolaldehyde (50-60\% yield), and formaldehyde ( $30-35 \%$ yield) [11, 13, 14]. A fourth product, 2-hydroxy-2-methylpropanal was also proposed. The identification of this product was based on: (1) The proposed reaction mechanism of $\mathrm{MBO}$ with $\cdot \mathrm{OH}$ (Figure 1) and a gas chromatographic (GC) retention index for the 2,4-dinitrophenylhydrazine (DNPH) derivative [13]; (2) identification of an ion in the chemical ionization mass spectra that corresponds to the $\left(\mathrm{M}-\mathrm{H}_{2} \mathrm{O}\right)^{+}$ion of a $\mathrm{C}_{4}$-hydroxycarbonyl or a $\mathrm{C}_{3}$-oxo-acid DNPH derivative [13]; and (3) identification of absorption bands corresponding to $\mathrm{C}-\mathrm{H}, \mathrm{C}=\mathrm{O}$, and $\left(\mathrm{CH}_{3}\right)_{3} \mathrm{Si}-\mathrm{OR}$ stretches in the GC-infrared spectrum and an ion corresponding to $\left(\mathrm{M}-\mathrm{CH}_{3}\right)^{+}$in the electron ionization MS of a trimethylsilyl derivatized sample extract [14]. The yield is assumed to be equal to that of formaldehyde [11]. However, Alvarado et al. [14] estimated a yield of $19 \pm$ $7 \%$, compared to the formaldehyde yield of $29 \pm 3 \%$. Although experimental evidence indicates a $\mathrm{C}_{4}$-hydroxycarbonyl, confirmation of 2-hydroxy-2-methylpropanal was not possible due to the absence of an authentic standard. Further, no ambient air data exists that relates concentrations of 2-hydroxy-2-methylpropanal to $\mathrm{MBO}$ emissions. The lack of information regarding the atmospheric generation and fate of 2-hydroxy-2-methylpropanal is in part due to the absence of a suitable analytical method to identify and quantify this compound in air.

We recently developed a method using a mist chamber for collection of compounds with a Henry's law constant $\geq 10^{3} \mathrm{M}$ atm $^{-1}$ [15]. O-(2,3,4,5,6-pentafluorobenzyl)hydroxylamine (PFBHA) in combination with bis-(trimethylsilyl) trifluoroacetamide (BSTFA) were used to derivatize carbonyl and hydroxyl/carboxyl groups, respectively. Detection and quantification were accomplished using gas chromatography/ion trap mass spectrometry (GC/ITMS) [16-20]. The power of ion trap mass spectrometry to facilitate identification of carbonyl and multifunctional carbonyl derivatives was presented in previous studies [18-20]. Herein, we describe the utility of ion trap mass spectrometry to identify 2-hydroxy-2methylpropanal in chamber studies and in air sampled in the Blodgett Forest. We confirm the identification by comparing the mass spectrum and GC retention time of the analyte in an extract of air to that of an authentic standard. In addition, we provide a method for semiquantification of 2-hydroxy-2-methylpropanal in air. An authentic standard is not commercially available, and hence other researchers who have not synthesized an authentic standard can use the method.

\section{Experimental}

\section{Gas Chromatography/Ion Trap Mass Spectrometry}

A Varian Star 3400 CX gas chromatograph (GC), with a temperature-programmable injector port interfaced to a Saturn 2000 ion trap mass spectrometer (Varian Associates, Walnut Creek, CA) was employed. Gas chromatographic separation was accomplished by using a capillary column coated with either (50\% phenylmethyl) polysiloxane (DB-17ms) or a formula with equivalent polarity to (12\% phenylmethyl) polysiloxane (DB-XLB). The dimensions of the columns were $30 \mathrm{~m} \times$ $0.25 \mathrm{~mm}$ i.d., $0.25 \mu \mathrm{m}$ film thickness (J\&W Scientific, Inc., Folsom, CA). A $5 \mathrm{~m}$ integrated guard column was used prior to the DB-XLB analytical column. The injector temperature was held at $280{ }^{\circ} \mathrm{C}$ for $1 \mathrm{~min}$, increased to $310^{\circ} \mathrm{C}$ at a rate of $50{ }^{\circ} \mathrm{C} \mathrm{min}{ }^{-1}$, held at $310^{\circ} \mathrm{C}$ for 25 min, then decreased to $280{ }^{\circ} \mathrm{C}$. The column temperature was held at $70{ }^{\circ} \mathrm{C}$ for $1 \mathrm{~min}$, increased to $100{ }^{\circ} \mathrm{C}$ at $5^{\circ} \mathrm{C}$ 
$\min ^{-1}$, increased to $280^{\circ} \mathrm{C}$ at $10^{\circ} \mathrm{C} \mathrm{min}^{-1}$, increased to $310^{\circ} \mathrm{C}$ at $30^{\circ} \mathrm{C} \mathrm{min}^{-1}$, then held at $310^{\circ} \mathrm{C}$ for $10 \mathrm{~min}$. The injection volume was $2 \mu \mathrm{L}$.

For the electron-ionization (EI), methane chemical ionization (CI), and pentafluorobenzyl alcohol $(\mathrm{PFBOH}) \mathrm{CI}$ mass spectra, we employed a filament current of $10 \mu \mathrm{A}$ and an ion trap temperature of $150{ }^{\circ} \mathrm{C}$. The EI mass spectra were acquired by scanning from $\mathrm{m} / \mathrm{z} 65$ to 650 , using automatic gain control with a target total ion current (TIC) of 20,000 and a maximum ionization time of $25,000 \mu \mathrm{s}$. We acquired the methane CI mass spectra by scanning from $\mathrm{m} / \mathrm{z} 150$ to 550, using automatic reaction control with a target TIC of 5000, a maximum ionization time of $500 \mu \mathrm{s}$, and a maximum reaction time of $80 \mathrm{~ms}$. PFBOH was introduced into the ion trap as described by Frazey et al. [19]. The PFBOH CI mass spectra were acquired by scanning from $\mathrm{m} / \mathrm{z}$ 230 to $m / z 650$, using automatic reaction control with a target TIC of 5000, a maximum ionization time of 2000 $\mu \mathrm{s}$, and a maximum reaction time of $128 \mathrm{~ms}$.

\section{Reagents and Glassware}

HPLC grade water (Fisher Scientific, Fairlawn, NJ) was purified by distillation in glass in the presence of $\mathrm{KMnO}_{4}$ to oxidize any organic contaminants. Other solvents were purchased in the highest purity available $(\geq 99.9 \%)$, and further purified by distillation in glass. O-(2,3,4,5,6-pentafluorobenzyl) hydroxylamine hydrochloride (PFBHA) ( $\geq 98 \%$ purity), O-(2,3,4,5,6-pentafluorobenzyl) alcohol (PFBOH) ( $\geq 98 \%$ purity), trimethylchlorosilane (TMCS), and carbonyl standards ( $\geq 95 \%$ purity) were purchased from Aldrich Chemical Company, Inc (Milwaukee, WI). Bis-(trimethylsilyl) trifluoroacetamide (BSTFA) was purchased from Supelco Chromatography Products (Bellefonte, PA). The PFBHA was recrystallized two times in 2-propanol. Other chemicals were used without further purification.

All glassware was soaked overnight in 15\% (vol/vol) dichlorodimethylsilane in toluene. The glassware was then rinsed with toluene, methanol, and dichloromethane (three times for each solvent), and dried at $150{ }^{\circ} \mathrm{C}$.

\section{PFBHA and BSTFA Derivatization}

PFBHA derivatization using a $1 \mathrm{mM}$ aqueous PFBHA solution was allowed to proceed for $24 \mathrm{~h}$ at room temperature. The PFBHA derivatives were extracted with dichloromethane. Two drops of concentrated $\mathrm{H}_{2} \mathrm{SO}_{4}$ and $5 \mathrm{~mL}$ of water were added to the sample extract to remove excess PFBHA [21], and the organic fraction was filtered through a $70 \mathrm{~mm} \times 7 \mathrm{~mm}$ o.d. column of anhydrous $\mathrm{Na}_{2} \mathrm{SO}_{4}(\mathrm{~s})$ to remove water. The eluate was collected and evaporated under a stream of $\mathrm{N}_{2}(\mathrm{~g})$ to a final volume of $2.0 \mathrm{~mL}$ for chamber studies, and $200 \mu \mathrm{L}$ for ambient air studies. Each time a vial was opened to add reagent or transfer the extract to another vial, the headspace was purged with $\mathrm{N}_{2}(\mathrm{~g})$ before it was sealed. Hydroxyl and carboxyl groups were derivatized with BSTFA containing 10\% trimethylchlorosilane (TMCS). Twenty $\mu \mathrm{L}$ of BSTFA:TMCS (90:10, v/v) was added to a vial containing $200 \mu \mathrm{L}$ of the PFBHAderivatized sample extract, and the vial was sealed with a teflon-coated cap. The BSTFA was allowed to react for 12 hours at $42{ }^{\circ} \mathrm{C}$, after which the derivatives were analyzed by GC/ITMS.

\section{Semi-Quantification of 2-Hydroxy-2-Methylpropanal}

Semi-quantification of 2-hydroxy-2-methylpropanal in ambient air samples was accomplished by using 3-hydroxy-3-methyl-2-butanone standard as a surrogate and 4-hydroxybenz $-{ }^{13} \mathrm{C}_{6}$-aldehyde as the internal standard. The analyses were conducted by using this method because an authentic standard of 2-hydroxy-2-methylpropanal was not available at the time when the analyses were conducted. We were unable to retroactively conduct such analyses because of concerns about degradation of the analyte in the sample extract. We employed 3-hydroxy-3-methyl-2-butanone as the surrogate because it is a carbonyl with a tertiary $-\mathrm{OH}$ group. The standard solutions used to construct the calibration curve contained 46 to $5500 \mathrm{pg} / \mu \mathrm{L}(0.451-53.9 \mu \mathrm{M})$ of 3-hydroxy-3-methyl-2-butanone. The concentration of the internal standard was $1045 \mathrm{pg} / \mu \mathrm{L}(8.16 \mu \mathrm{M})$. Eightor 10-point calibration curves were constructed by the analysis of four or five standard solutions before and after analysis of samples. Recoveries of 3-hydroxy-3methyl-2-butanone were obtained by the analysis $1 \mathrm{mM}$ aqueous PFBHA solutions enriched with $185 \mathrm{ng}$ (1.81 $\mathrm{mmol}$ ) of 3-hydroxy-3-methyl-2-butanone at the time of ambient air sample collection (field spikes). The recoveries of the field spikes for the chamber $(n=2)$ and ambient air $(n=2)$ experiments were 90 and $98 \%$, and 113 and 91\%, respectively. A mid-point calibration standard (231 or $461 \mathrm{pg} / \mu \mathrm{L} ; 2.26$ or $4.52 \mu \mathrm{M}$ ) was analyzed after every five samples. The relative standard deviation among the relative response factors for standard curves was $\leq 28 \%$.

\section{Blanks and Limit of Detection (LOD)}

For chamber studies, air samples collected from the chamber in impingers before addition of $\mathrm{MBO}, \mathrm{NO}$, and $\mathrm{NO}_{2}$, were used as blanks. These blanks were treated identically to samples. For ambient air studies, ultrahigh purity (UHP) zero grade air was sampled by using a mist chamber to determine background concentrations of the carbonyls. These samples were employed as blanks, and treated identically to samples. Carbonyl compounds are ubiquitous in air, solvents, and many reagents. Extensive purification procedures, treatment of glassware to reduce contamination, reducing exposure of glassware, and samples to ambient air, signifi- 
cantly reduce the background concentration, but do not remove carbonyls entirely. We used a standard method for calculating the LOD, which considers the variability among blank samples. The LOD was calculated as $3 \sigma_{\text {blnk }}$, where $\sigma_{\text {blnk }}$ is the standard deviation of the response factor for the analyte in three or more reagent blanks [22]. The analyte concentration in the sample was considered significant only if it exceeded the LOD after subtraction of the mean blank concentration.

\section{$\mathrm{OH}$ Radical Oxidation of 2-Methyl-3-Buten-2-Ol in Chambers}

Alvarado et al. [14] previously described conditions utilized in the chamber studies. Briefly, a 5800-liter evacuable, Teflon-coated chamber containing a multiple reflection optical system interfaced to a Nicolet 7199 Fourier transform infrared (FT-IR) spectrometer was employed. Irradiation was provided by a $24 \mathrm{~kW}$ xenon arc filtered through a Pyrex pane to remove wavelengths $<300 \mathrm{~nm}$. The experiment was conducted at $298 \pm 2 \mathrm{~K}$ and $749 \pm 2$ torr total pressure. Initial concentrations of reactants were $10 \mathrm{ppm}\left(2.4 \times 10^{14}\right.$ molecules $\mathrm{cm}^{-3}$ ) each, $\mathrm{MBO}, \mathrm{CH}_{3} \mathrm{ONO}$, and $\mathrm{NO} . \mathrm{OH}$ radicals were generated by photolysis of $\mathrm{CH}_{3} \mathrm{ONO}$ [23]. Three irradiations were performed for 1.5-2 min. During dark periods, samples were collected in two midget $(25 \mathrm{~mL})$ impingers in series, which each contained 20 $\mathrm{mL}$ of $1 \mathrm{mM}$ of aqueous PFBHA. Impinger samples were collected on ice for $10 \mathrm{~min}$ at an airflow rate of 113 $\mathrm{mL} \min ^{-1}$. Each sample was enriched with $100 \mu \mathrm{L} 20.1$ ng $\mu \mathrm{L}^{-1}(157 \mu \mathrm{M})$ 4-hydroxybenz- ${ }^{13} \mathrm{C}_{6}$-aldehyde after collection.

\section{Collection of Ambient Air Samples}

Ambient air samples were collected above a ponderosa pine plantation on August 17, 2000, between the hours of 6:00 and 21:00, local standard time (LST). The site is located in the western foothills of the Sierra Nevada, near the Blodgett Forest Research Station (38 $53^{\prime}, 42.9^{\prime \prime}$ $\mathrm{N}, 120^{\circ} 37^{\prime}, 57.9^{\prime \prime} \mathrm{W}, 1315 \mathrm{~m}$ elevation). The pine trees are even aged (9-10 years old), and planted at a density of 0.12 trees $\mathrm{m}^{-2}$, with various coniferous and deciduous trees interspersed. Detailed descriptions of the site can be found elsewhere [2, 24]. Typical daytime mixing ratios of $\mathrm{MBO}$ at this site during summer are 1-3 ppbv [24].

Ambient air samples were collected in a mist chamber, which was previously described [15, 25, 26]. For this work, air was sampled for 10 minutes in two mist chambers in series, at a flow rate of approximately 23-25 L min ${ }^{-1}$. The mist chamber reservoir was filled with $20 \mathrm{~mL}$ of $1 \mathrm{mM}$ aqueous PFBHA. Each sample was enriched with $50 \mu \mathrm{L}$ of $4.18 \mathrm{ng} \mu \mathrm{L}^{-1}(32.7 \mu \mathrm{M})$ 4-hydroxybenz $-{ }^{13} \mathrm{C}_{6}$-aldehyde after collection.

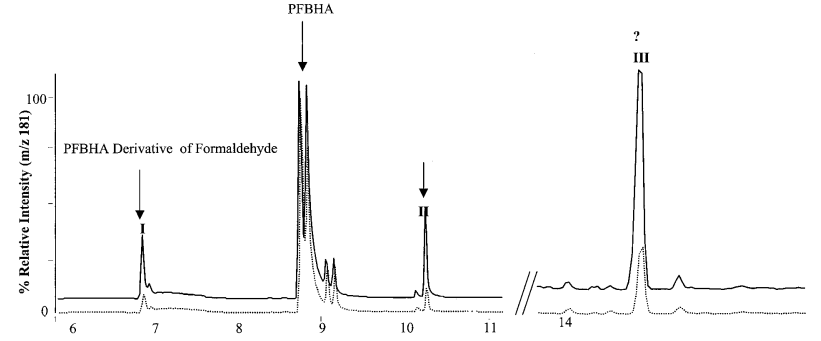

Figure 2. $m / z 181$ ion chromatogram of a PFBHA-BSTFA derivative of products arising from the reaction of $\mathrm{MBO}$ with $\cdot \mathrm{OH}$ in an environmental chamber (solid line: sample extract; dashed lines: blank extract)

\section{Synthesis of 2-Hydroxy-2-Methylpropanal}

The synthesis began with the straight-forward 1,2addition of methyl magnesium bromide in ether [27]. Despite several attempts, subsequent hydrolysis of the acetal in the presence of the secondary alcohol proved to be problematic, giving a variety of undesired side products and poor yields of the hydroxy aldehyde. Thus, to circumvent these problems the free alcohol was benzyl protected under standard conditions [28], followed by the acid catalyzed hydrolysis of the acetal to readily give aldehydes [29]. Then hydrogenation of the benzyl ether under standard conditions gave 2-hydroxy-2-methylpropanal [30].

\section{Results and Discussion}

\section{Identification of Products Arising from $\mathrm{OH}$ Radical Oxidation of $M B O$ in Chambers}

Previous research conducted in chambers establishes that hydroxyl radical oxidation of $\mathrm{MBO}$ yields acetone, glycolaldehyde, formaldehyde, and a $\mathrm{C}_{4}$-hydroxycarbonyl, tentatively identified as 2-hydroxy-2-methylpropanal $[11,13,14]$. Due to the lack of an authentic standard, the yield was not calculated and the identity of 2-hydroxy-2-methylpropanal (i.e., the structure of the $\mathrm{C}_{4}$-hydroxycarbonyl) was not confirmed.

Electron ionization (EI) of a PFBHA derivative of a carbonyl generates a pentafluorobenzyl cation $\left[\left(\mathrm{C}_{6} \mathrm{~F}_{5} \mathrm{CH}_{2}\right)^{+}\right]$which is $181 \mathrm{Da}[18,19,30]$. The presence of a $\mathrm{m} / \mathrm{z} 181$ ion in the EI mass spectra of PFBHA derivatives in a sample extract thus denotes the presence of a carbonyl. At least three carbonyl compounds are evident in the $\mathrm{m} / \mathrm{z} 181$ ion chromatogram of a sample (solid line) and blank (dashed line) extract of air collected from a chamber experiment in which MBO was reacted with $\cdot \mathrm{OH}$. These products are labeled I, II, and III in Figure 2. These compounds are significant reaction products because they are present at least $3 \times$ the peak area of background levels. By comparing the relative retention times and mass spectra from Peaks I and II to those of PFBHA derivatized standards, we confirmed the identities of formaldehyde (I) and ace- 


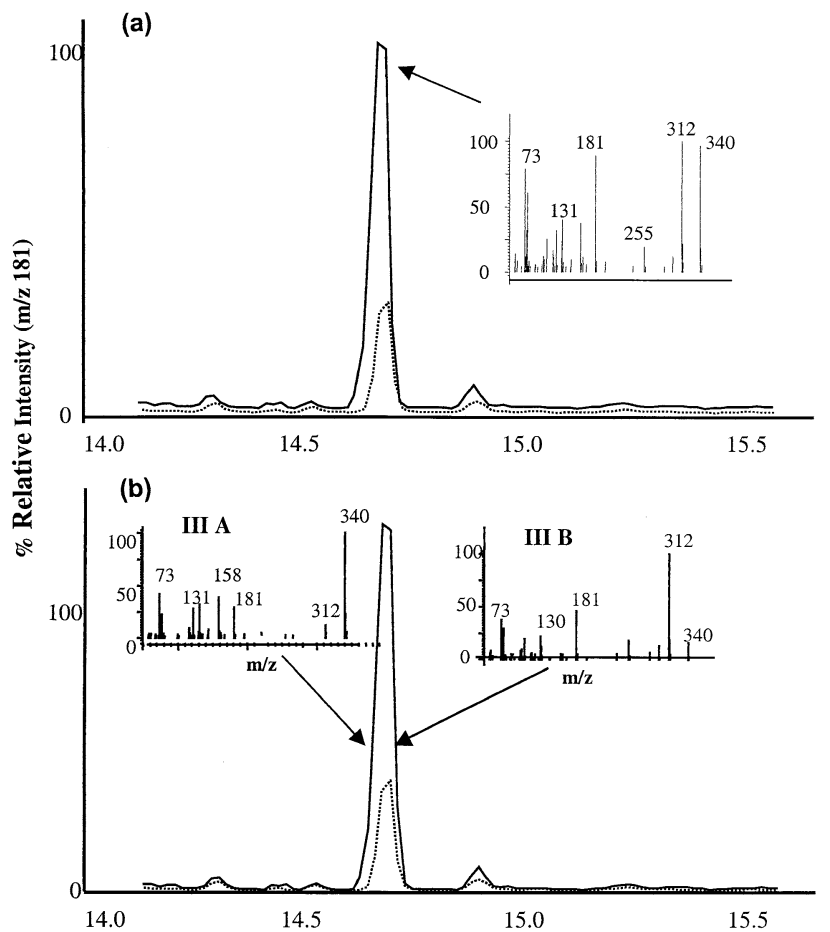

Figure 3. Electron ionization mass spectra of a PFBHA-BSTFA derivative of a photooxidation product(s) arising from the reaction of $\mathrm{MBO}$ with $\cdot \mathrm{OH}$ in an environmental chamber: Electron ionization mass spectrum at the centroid of Peak III (a); electron ionization mass spectra from the left and right sides of Peak III (b).

tone (II). The large $m / z 181$ peak that elutes around 9 min is due to excess PFBHA reagent.

The EI mass spectrum at the centroid of Peak III is presented in Figure 3a. The presence of ions at $\mathrm{m} / \mathrm{z} 73$, 75, and 181 indicate that the compound is a PFBHABSTFA derivative of a hydroxycarbonyl or an aldo- or keto-acid. The $m / z 73$ and 75 ions are $\left[\left(\mathrm{CH}_{3}\right)_{3} \mathrm{Si}\right]^{+}$and $\left[\mathrm{HO}=\mathrm{Si}\left(\mathrm{CH}_{3}\right)_{2}\right]^{+}$ions that arise from fragmentation of derivatized hydroxyl or carboxyl groups on the molecule. Examination of the mass spectra on the left and right sides of Peak III revealed the presence of two compounds, IIIA, with a base peak at $m / z 340$, and IIIB, with a base peak at $m / z 312$ (see Figure $3 b$ ). The base peak of a PFBHA-BSTFA hydroxycarbonyl derivative is generally due to an $(\mathrm{M}-15)^{+}$ion $[18,19]$. We thus hypothesized that the $(\mathrm{M}-15)^{+}$ions for Compounds IIIA and IIIB were $m / z 340$ and 312, respectively. This assumption led to the determination of molecular weights for the PFBHA-BSTFA derivatives of Compound IIIA and IIIB of 355 and $327 \mathrm{Da}$, respectively. We tentatively identified IIIB as the PFBHA-BSTFA derivative of glycolaldehyde, and confirmed the identity by comparing the relative retention time and the EI mass spectra to the PFBHA-BSTFA derivative of an authentic standard.

To assist in identifying the compound in Peak IIIA, we obtained the methane $\mathrm{CI}$ mass spectrum, presented in Figure 4a. The methane CI mass spectra of PFBHABSTFA derivatives of aliphatic hydroxycarbonyls are

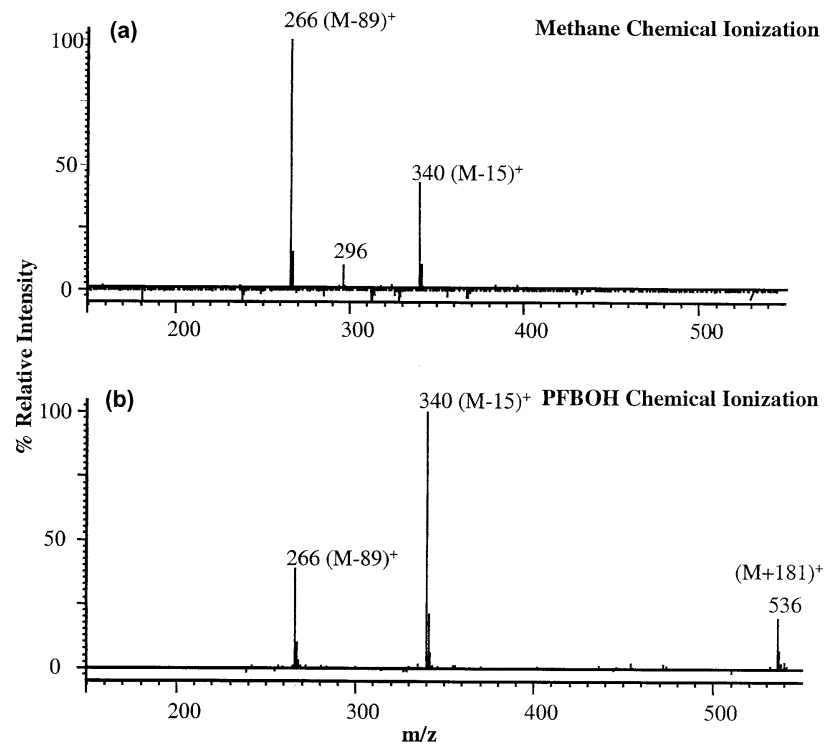

Figure 4. Methane chemical ionization (a) and PFBOH chemical ionization (b) mass spectra of the PFBHA-BSTFA derivative in Peak IIIA of Figure 3.

characterized by $(\mathrm{M}+1)^{+},(\mathrm{M}-15)^{+}$, and $(\mathrm{M}-89)^{+}$ ions, which are generated by addition of a proton, loss of $\left(\mathrm{CH}_{3}\right)$, and loss of [ $\left.\mathrm{Si}\left(\mathrm{CH}_{3}\right)_{3} \mathrm{O}\right]$, respectively [17-19]. If our hypothesis was correct (i.e., the molecular weight of the derivative in Peak IIIA is $355 \mathrm{Da}$ ), we would expect to observe ions at $m / z 356,340$, and 266. However, in this case, the base peak is at $m / z 266$, with a smaller peak at $m / z 340$, which could be the $(\mathrm{M}-15)^{+}$ ion. The absence of an ion at $\mathrm{m} / \mathrm{z} 356$ makes the identification of the molecular weight inconclusive.

In previous work, interpretation of the $\mathrm{PFBOH} \mathrm{CI}$ ion trap mass spectra was critical to the identification of compounds for which authentic standards do not exist (i.e., unknowns) $[18,19]$. The $(\mathrm{M}+181)^{+}$ion arises from ion-molecule reactions that occur in an ion trap mass spectrometer, but not in a conventional quadrupole mass spectrometer [19, 30-32]. The relationship between the $(\mathrm{M}+\mathrm{H})^{+}$and $(\mathrm{M}+181)^{+}$ions has proven invaluable in certain cases for identification of the molecular ion [18, 19]. In the PFBOH CI mass spectrum of Compound IIIA, we observe ions at $\mathrm{m} / \mathrm{z} 266,340$, and 536. (See Figure $4 \mathrm{~b}$ ). Because of the knowledge about the $\mathrm{CI}$ and PFBOH CI mass spectra of PFBHA-BSTFA derivatives attained in previous studies [18-20], we can assume that the ion at $m / z 536$ is the $(\mathrm{M}+181)^{+}$ion of a compound with a molecular weight of $355 \mathrm{Da}$. The absence of an $(\mathrm{M}+\mathrm{H})^{+}$ion at $m / z 356$ is inconsistent with previously observations regarding the $\mathrm{PFBOH} \mathrm{CI}$ mass spectra of PFBHA-BSTFA derivatives of multifunctional carbonyls, and indicates that the formation of a stable $(\mathrm{M}+\mathrm{H})^{+}$ion might be dependent on the structure of the molecule. A molecular weight of $355 \mathrm{Da}$ for the PFBHA-BSTFA derivative indicates the presence of a $\mathrm{C}_{4}$-hydroxycarbonyl or a $\mathrm{C}_{3}$-oxo-acid. The gold 
Table 1. Characterization of the electron ionization, methane chemical ionization, and $\mathrm{PFBOH}$ chemical ionization mass spectra for PFBHA-BSTFA derivatives of carbonyls with primary, secondary, and tertiary - OH groups

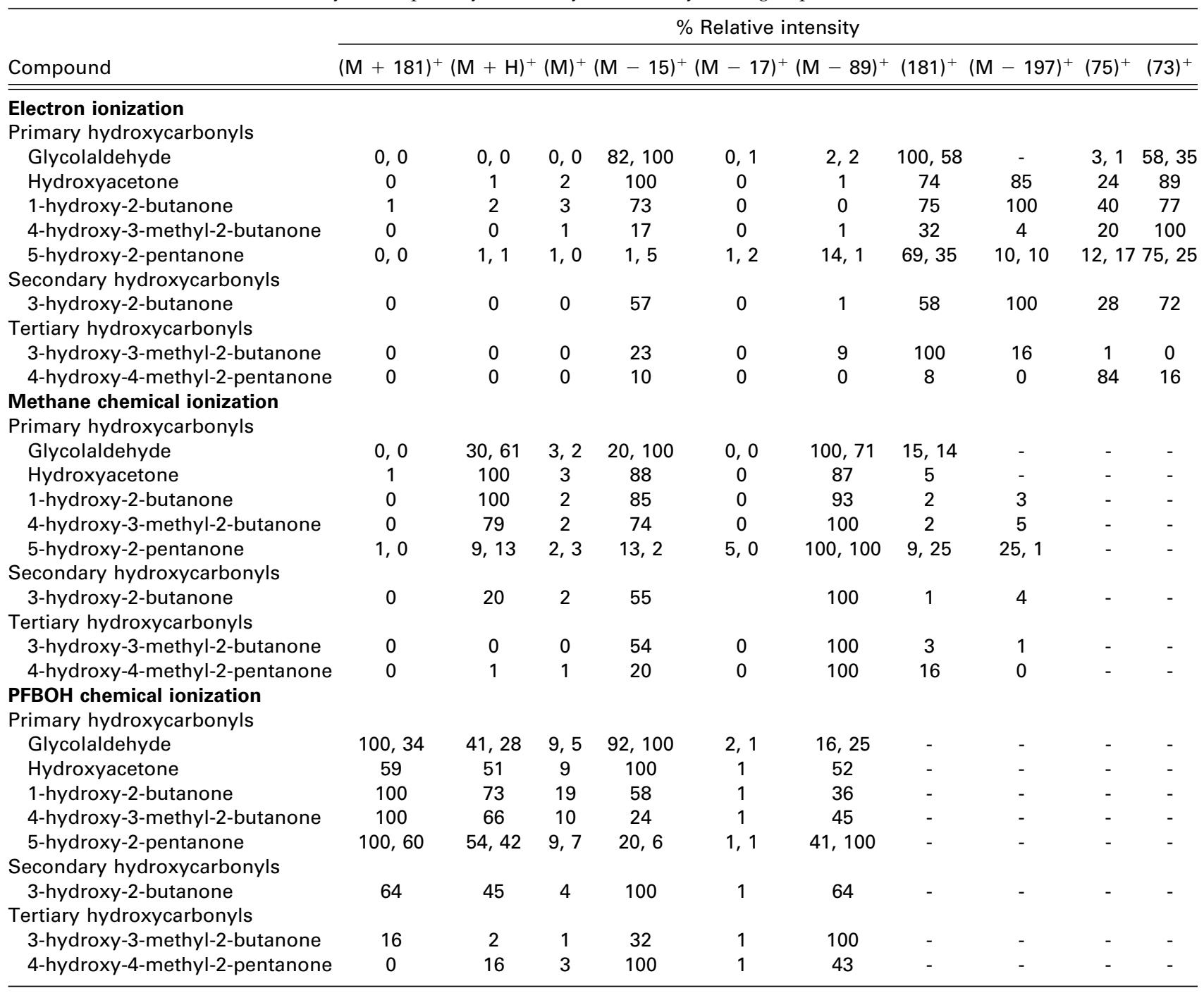

standard to distinguish whether the compound was a hydroxycarbonyl or an oxo-acid is to obtain an accurate mass measurement on the molecular or pseudo-molecular ion. In this case, the experiment would need to be conducted at a resolving power $=10,000$ under methane chemical ionization conditions. Under CI conditions, the relative intensity of the $(\mathrm{M}+\mathrm{H})^{+}$ion of PFDHA/BSTFA derivatives is compound dependent and may exist at low intensities, thereby making it difficult to conduct an accurate mass measurement on the ion as it elutes from the GC. Herein, we utilize an approach that relies on knowledge of the mass spectrometry of PFBHA-BSTFA derivatives of aldo- and ketoacids, and hydroxycarbonyls to derive the elemental formula of the compound in Peak IIIA. One advantage of our approach is that it relies on instrumentation that is less expensive than double-focusing mass spectrometers, which are typically employed to perform accurate mass measurements.

\section{Characterization of the EI, Methane CI, and PFBOH CI Mass Spectra of Aldo- and Keto-Acids and Carbonyls with Primary, Secondary, and Tertiary - OH Groups}

The indication that the generation of the $(\mathrm{M}+\mathrm{H})^{+}$ion may be dependent on the structure of the PFBHABSTFA derivative led us to examine the EI, methane CI, and PFBOH CI mass spectra of PFBHA-BSTFA derivatives of aldo- and keto-acid carbonyls with primary, secondary, and tertiary $-\mathrm{OH}$ groups. The EI mass spectra of PFBHA-BSTFA derivatives of carbonyls with primary and secondary - $\mathrm{OH}$ groups and aldo- and keto-acids are characterized by ions at $m / z 73,75,181$, $(\mathrm{M}-15)^{+}$, and $(\mathrm{M}-197)^{+}$. (See Tables 1 and 2$)$. The base peak in the EI mass spectra of the compounds investigated is either the $(\mathrm{M}-15)^{+}$ion, the $(\mathrm{M}-197)^{+}$ ion, $m / z 181$, or $m / z$ 73. For the hydroxycarbonyls, a consistent trend that can be used to identify substitution 
Table 2. Characterization of the electron ionization, methane chemical ionization, and $\mathrm{PFBOH}$ chemical ionization mass spectra for PFBHA-BSTFA derivatives of aldo- and keto-acids

\begin{tabular}{|c|c|c|c|c|c|c|c|c|c|c|}
\hline \multirow[b]{2}{*}{ Compound } & \multicolumn{10}{|c|}{$\%$ Relative intensity } \\
\hline & $(M+181)^{+}$ & $(\mathrm{M}+\mathrm{H})^{+}$ & $(\mathrm{M})^{+}$ & $(\mathrm{M}-15)^{+}$ & $(\mathrm{M}-17)^{+}$ & $(\mathrm{M}-89)^{+}$ & $(181)^{+}$ & $(\mathrm{M}-197)^{+}$ & $(75)^{+}$ & $(73)^{+}$ \\
\hline \multicolumn{11}{|l|}{ Electron ionization } \\
\hline \multicolumn{11}{|l|}{ Aldo-acids } \\
\hline Glyoxylic acid & 2 & 2 & 8 & 36 & 1 & 0 & 100 & 1 & 6 & 41 \\
\hline \multicolumn{11}{|l|}{ Keto-acids } \\
\hline Pyruvic acid & 1 & 2 & 9 & 23 & 19 & 1 & 100 & 0 & 6 & 54 \\
\hline 2-ketobutyric acid & 0,0 & 2,2 & 10,7 & 13,28 & 46,45 & 0,1 & 100,100 & 0,1 & 16,13 & 97,83 \\
\hline 2-oxovaleric acid & 1,2 & 4,8 & 11,11 & 11,51 & 54,52 & 1,12 & 100,100 & 19,20 & 12,21 & 98,92 \\
\hline \multicolumn{11}{|c|}{ Methane chemical ionization } \\
\hline \multicolumn{11}{|l|}{ Aldo-acids } \\
\hline Glyoxylic acid & 19 & 100 & 1 & 43 & 1 & 15 & 17 & - & - & - \\
\hline \multicolumn{11}{|l|}{ Keto-acids } \\
\hline Pyruvic acid & 1 & 100 & 5 & 25 & 1 & 28 & 4 & 1 & - & - \\
\hline 2-ketobutyric acid & 0,1 & 28,100 & 0,1 & 3,21 & 3,1 & 9,100 & 100,17 & 11,1 & - & - \\
\hline 2-oxovaleric acid & - & 100,100 & 3,2 & 3,25 & 4,0 & 0,19 & 52,4 & 0,7 & - & - \\
\hline \multicolumn{11}{|c|}{$\begin{array}{l}\text { PFBOH chemical ionization } \\
\text { Aldo-acids }\end{array}$} \\
\hline Glyoxylic acid & 100 & 21 & 7 & 22 & 0 & 8 & - & - & - & - \\
\hline \multicolumn{11}{|l|}{ Keto-acids } \\
\hline Pyruvic acid & 100 & 66 & 37 & 30 & 23 & 15 & - & - & - & - \\
\hline 2-ketobutyric acid & 100,91 & 35,31 & 0,19 & 45,68 & 21,7 & 0,27 & - & - & - & - \\
\hline 2-oxovaleric acid & 100,100 & 20,43 & 8,4 & 7,37 & 9,7 & 0,5 & & & & \\
\hline
\end{tabular}

of the $-\mathrm{OH}$ group is not evident. However, the presence of an $(\mathrm{M})^{+\cdot}$ ion ranging from 7 to $11 \%$ relative intensity is unique to the PFBHA-BSTFA derivatives of the aldo- and keto-acids, and the presence of an ( $\mathrm{M}$ $17)^{+}$ion ranging from 19 to $52 \%$ relative intensity is unique to the PFBHA-BSTFA derivatives of the ketoacids $\left[(\mathrm{M})^{+\cdot}\right.$ and $(\mathrm{M}-17)^{+}$ions are $\leq 3 \%$ for hydroxycarbonyls]. The $(\mathrm{M})^{+\cdot}$ ion of a PFBHA-BSTFA derivative of an aldo- or keto-acid is stabilized by charge delocalization, as depicted in Figure 5a. The $(\mathrm{M}-17)^{+}$ion likely results from rearrangement and loss of $\mathrm{OH}$ from the derivatized acid. Because the $(\mathrm{M}-17)^{+}$ion is not observed in the mass spectrum of the aldo-acid, we assume that the alkyl group $\left(\mathrm{CHR}_{2}\right.$ in Figure $\left.5 \mathrm{~b}\right)$ is essential in the rearrangement $\left(\mathrm{CHR}_{2}=\mathrm{H}\right.$ for an aldoacid). The proposed fragmentation/rearrangement pathway leading to the formation of an $(\mathrm{M}-17)^{+}$ion is depicted in Figure $5 \mathrm{~b}$. Thus, the presence of a lowintensity $(\mathrm{M})^{+\cdot}$ ion in the EI mass spectrum suggests an aldo- or keto-acid, while the presence of an $(\mathrm{M}-17)^{+}$ ion suggests a keto-acid.

The methane CI mass spectra of PFBHA-BSTFA derivatives of aldo- and keto-acids, and hydroxycarbonyls are characterized by $(\mathrm{M}+\mathrm{H})^{+},(\mathrm{M}-15)^{+}$, and $(\mathrm{M}-89)^{+}$ions. (See Tables 1 and 2). For the carbonyls with secondary and tertiary $-\mathrm{OH}$ groups, the base peak in the mass spectra is the $(\mathrm{M}-89)^{+}$ion, whereas for the carbonyls with primary $-\mathrm{OH}$ groups and the aldo- and keto-acids, the base peak can be the $(\mathrm{M}+\mathrm{H})^{+}$ion, the $(\mathrm{M}-15)^{+}$ion, or the $(\mathrm{M}-89)^{+}$ion. The relative intensity of the $(\mathrm{M}+\mathrm{H})^{+}$ion from the carbonyls with primary and secondary $-\mathrm{OH}$ groups ranges from 9$100 \%$. For the aldo- and keto-acids, the relative intensity of the $(\mathrm{M}+\mathrm{H})^{+}$ion ranges from $28-100 \%$. However, the relative intensity of this ion for the carbonyls with tertiary $-\mathrm{OH}$ groups was $\leq 1 \%$. While there is no absolute trend in these data, the presence of the $(\mathrm{M}-$ $89)^{+}$ion as the base peak, and a low intensity $(\mathrm{M}+\mathrm{H})^{+}$ ion $(\leq 1 \%)$ in the methane CI mass spectra suggests a
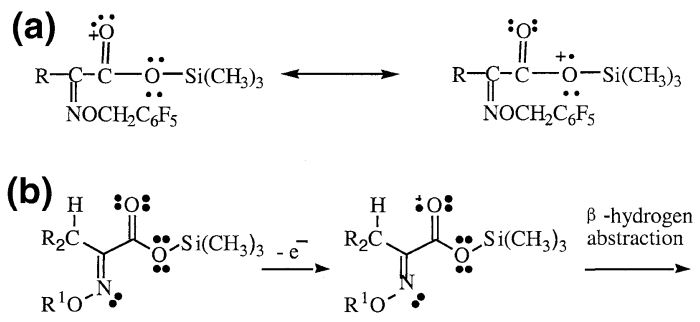
PFBHA-BSTFA derivative $\mathrm{M}^{+\bullet}$ $\left(\mathrm{R}^{1}=\mathrm{CH}_{2} \mathrm{C}_{6} \mathrm{~F}_{5}\right)$

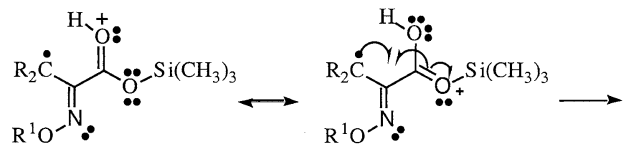

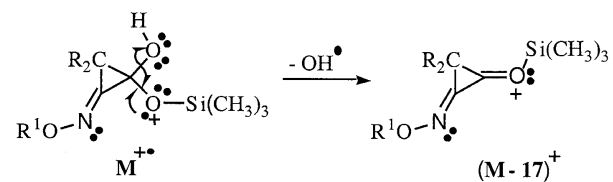

Figure 5. Proposed fragmentation pathway of PFBHA-BSTFA derivatives of aldo- and keto-acids under electron ionization conditions in an ion trap mass spectrometer ( $\mathrm{R}=\mathrm{H}$ or alkyl). (a) Proposed mechanism for stabilization of the PFBHA-BSTFA derivative of an aldo- or keto-acid. (b) Proposed pathway for the formation of an $(\mathrm{M}-17)^{+}$ion from a PFBHA-BSTFA derivative of a keto-acid. 
PFBHA-BSTFA derivative of a carbonyl with a tertiary $-\mathrm{OH}$ group.

The PFBOH CI ion trap mass spectra of PFBHABSTFA derivatives are characterized by $(\mathrm{M}-89)^{+}$, $(\mathrm{M}-15)^{+},(\mathrm{M}+\mathrm{H})^{+}$, and $(\mathrm{M}+181)^{+}$ions. For most of the carbonyls with primary and secondary $-\mathrm{OH}$ groups and the aldo- and keto-acids, the base peak is either the $(\mathrm{M}+181)^{+}$ion or the $(\mathrm{M}-15)^{+}$ion. For the carbonyls with tertiary $-\mathrm{OH}$ groups, the base peak is either the $(\mathrm{M}-15)^{+}$ion or the $(\mathrm{M}-89)^{+}$ion. For these carbonyls, both the $(\mathrm{M}+\mathrm{H})^{+}$ion and the $(\mathrm{M}+181)^{+}$ion are of low $(\leq 16 \%)$ relative intensity in the $\mathrm{PFBOH} \mathrm{CI}$ mass spectra, whereas the $(\mathrm{M}+\mathrm{H})^{+}$ion in the PFBOH CI mass spectra of the carbonyls with primary and secondary $-\mathrm{OH}$ groups and the aldo- and keto-acids ranges from $20-73 \%$ relative intensity and the $(M+181)^{+}$ion ranges from $34-100 \%$ relative intensity. Accordingly, a base peak at $(\mathrm{M}-15)^{+}$or $(\mathrm{M}-89)^{+}$and a low relative intensity for the $(\mathrm{M}+\mathrm{H})^{+}$and $(\mathrm{M}+181)^{+}$ions in PFBOH CI $(\leq 16 \%)$ indicates a PFBHA-BSTFA derivative of a carbonyl with a tertiary $-\mathrm{OH}$ group.

These data suggest that interpretation of the EI mass spectra can assist in distinguishing PFBHA-BSTFA derivatives of aldo- and keto-acids from derivatives of hydroxycarbonyls, and that interpretation of the methane $\mathrm{CI}$ and $\mathrm{PFBOH} \mathrm{CI}$ mass spectra can assist in distinguishing the position of the $-\mathrm{OH}$ group on hydroxycarbonyls. In the EI mass spectra, an $(\mathrm{M})^{+\cdot}$ ion with a relative intensity $\geq 7 \%$ indicates the PFBHA-BSTFA derivative of an aldo- or keto-acid, while an $(\mathrm{M}-17)^{+}$ion with a relative intensity $\geq 19 \%$ indicates a keto-acid. In the methane CI mass spectra, a base peak at $(\mathrm{M}-89)^{+}$and a low intensity $(\leq 1 \%)(\mathrm{M}+\mathrm{H})^{+}$ion strongly suggests a carbonyl with a tertiary $-\mathrm{OH}$ group. The identification of a carbonyl with a tertiary $-\mathrm{OH}$ group is strengthened by a base peak at $(\mathrm{M}-15)^{+}$or $(\mathrm{M}-89)^{+}$and low intensity $(\leq 16 \%)(\mathrm{M}+\mathrm{H})^{+}$and $(\mathrm{M}+181)^{+}$ions in the PFBOH CI ion trap mass spectra.

The EI mass spectrum for the PFBHA-BSTFA derivative from the $\mathrm{MBO}+\cdot \mathrm{OH}$ reaction (Figure 3, Compound IIIA) provides evidence that this compound is a $\mathrm{C}_{4}$-hydroxycarbonyl, rather than a $\mathrm{C}_{3}$-aldo- or keto-acid because $\mathrm{M}^{+\cdot}$ and $(\mathrm{M}-17)^{+}$ions are absent. The methane $\mathrm{CI}$ and $\mathrm{PFBOH} \mathrm{CI}$ mass spectra (Figure 4) provide substantial evidence that the $-\mathrm{OH}$ group is tertiary, because of the absence of an $(\mathrm{M}+\mathrm{H})^{+}$ion in both spectra and the low intensity of the $(\mathrm{M}+181)^{+}$ion in the PFBOH CI mass spectrum. The only possible $\mathrm{C}_{4}{ }^{-}$ hydroxycarbonyl containing a tertiary $-\mathrm{OH}$ group is 2-hydroxy-2-methylpropanal.

Gas Chromatographic Separation of the PFBHA-BSTFA Derivatives of Glycolaldehyde, 2-Hydroxy-2-Methylpropanal, and 3-Hydroxy-2-Butanone

Extracts of air sampled at the Blodgett Forest were first analyzed by using a low-polarity GC column [DB-XLB;
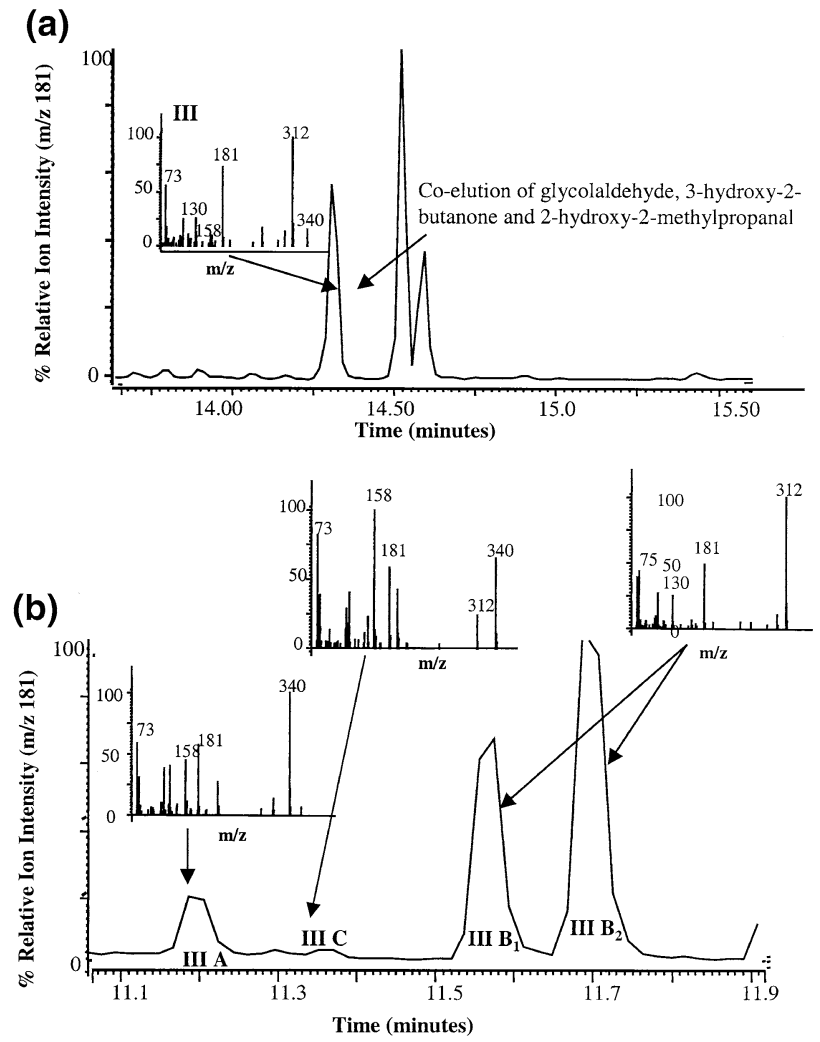

Figure 6. Chromatographic separation of PFBHA-BSTFA derivatives in an extract of Blodgett Forest air on a DB-XLB GC column (a), and a DB-17ms GC column (b).

liquid phase equivalent to ( $12 \%$ phenylmethyl) polysiloxane]. The electron ionization mass spectrum of a peak from these extracts with the same relative retention time as the Peak III in Figure 2 is presented in Figure 6. As determined previously, this peak can be composed of two co-eluting compounds, glycolaldehyde and 2-hydroxy-2-methylpropanal, both of which are expected to be present in Blodgett Forest air. The $\mathrm{m} / \mathrm{z} 312$ ion is likely the $(\mathrm{M}-15)^{+}$ion for PFBHABSTFA derivatized glycolaldehyde, and the $\mathrm{m} / \mathrm{z} 340$ ion is likely the $(\mathrm{M}-15)^{+}$ion for PFBHA-BSTFA derivatized 2-hydroxy-2-methylpropanal. However, it is possible that this peak could also contain 3-hydroxy-2butanone, which we identified at a similar relative retention time in previous work [18]. In this complex mixture, quantification of glycolaldehyde is possible by using the $(\mathrm{M}+\mathrm{H})^{+}$ion at $m / z 328$ in the methane CI mass spectra. However, quantifying 2-hydroxy-2-methylpropanal in the presence of 3-hydroxy-2-butanone poses a problem because the two compounds are structural isomers whose mass spectra would exhibit ions at the same mass to charge ratios. Additionally, in order to confirm the identification of 2-hydroxy-2-methylpropanal with an authentic standard, chromatographic resolution is desired. We thus pursued separation of these compounds on a GC column with a more polar phase, [( $50 \%$ phenylmethyl) polysiloxane; DB- $17 \mathrm{~ms}]$.

We demonstrate resolution of three co-eluting com- 

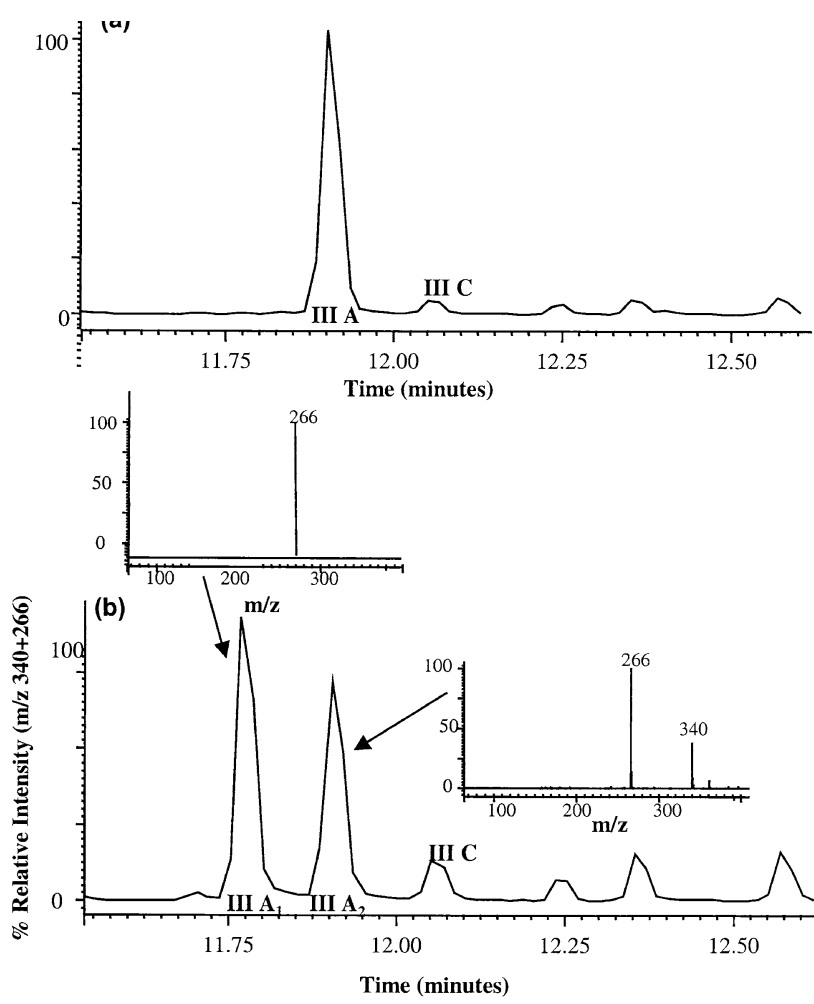

Figure 7. Ion chromatogram $(m / z 266+m / z 340)$ in the methane chemical ionization mass spectra of PFBHA-BSTFA derivatives in an extract of Blodgett Forest air.

pounds on a DB-17ms GC column in Figure 6b. The mass spectrum and relative retention time of Compound IIIA corresponds to the mass spectrum and relative retention time of the PFBHA-BSTFA derivative that was obtained from the analysis of the reaction products of hydroxyl radical reaction with MBO (see Figure $3 \mathrm{~b}$ Compound IIIA). The compounds in Peaks IIIB $_{1}$ and IIIB $_{2}$ correspond to the mass spectra of glycolaldehyde (see Figure 3b, Compound IIIB). The compound in Peak IIIC was tentatively identified as 3hydroxy-2-butanone. The identification of the PFBHABSTFA derivatives of glycolaldehyde and 3-hydroxy-2butanone were confirmed by comparing the relative retention times and mass spectra in the sample extract to those of the PFBHA-BSTFA derivatives of authentic standards (Peaks IIIB $_{1}$ and IIIB $_{2}$ are the $E$ - and Zisomers of the PFBHA-BSTFA derivative of glycolaldehyde; the $E$ - and Z-isomers of 3-hydroxybutanone are not resolved).

In the methane $\mathrm{CI}$ ion chromatograms comprised of $\mathrm{m} / \mathrm{z} 340$ and 266 , the $(\mathrm{M}-15)^{+}$and $(\mathrm{M}-89)^{+}$ions of the PFBHA-BSTFA derivative of 2-hydroxy-2-methylpropanal, we observed two peaks corresponding to 3-hydroxy-2-butanone (IIIC) and the compound tentatively identified as 2-hydroxy-2-methylpropanal (IIIA) (see Figure 7a) in some sample extracts. However, in other extracts, we observed three peaks (IIIA , IIIA $_{2}$, and IIIC), as presented in Figure $7 \mathrm{~b}$. Moreover, the relationship between Peaks IIIA I $_{1}$ and IIIA $_{2}$ was not constant. In some cases, Peak IIIA 1 was larger than
Peak IIIA ${ }_{2}$, and in other cases, the peaks were approximately the same size, or Peak IIIA $\mathbf{A}_{2}$ was absent. To further investigate this phenomenon, we reanalyzed extracts of air collected from the reaction of $\mathrm{MBO}$ with $\cdot \mathrm{OH}$ in the chamber experiment, using the DB- $17 \mathrm{~ms}$ column. We observed the same phenomenon in these extracts. Thus, the compounds comprising Peaks IIIA and IIIA $\mathbf{A}_{2}$ must be products arising from the reaction of $\mathrm{MBO}$ with $\cdot \mathrm{OH}$.

The base peak in the methane chemical ionization mass spectra of Peaks IIIA $\mathbf{A}_{1}$ and IIIA $\mathbf{A}_{2}$ is the $m / z 266$ ion. Initially, we thought that the two peaks were the $E$ - and Z-isomers of PFBHA-BSTFA derivatives of 2-hydroxy-2-methylpropanal. However, the absence of an $(\mathrm{M}-15)^{+}$ion at $m / z 340$ in Peak IIIA $_{1}$ raised doubt about this identification. The ion at $\mathrm{m} / \mathrm{z} 266$ in Peak IIIA $_{1}$ could be the $(\mathrm{M}-89)^{+}$ion of the PFBHA-BSTFA derivative of 2-hydroxy-2-methylpropanal, or it could be the $(\mathrm{M}+\mathrm{H})^{+}$ion or a fragment ion from a different compound. We examined the EI and PFBOH CI mass spectra to obtain further information.

The EI and PFBOH CI mass spectra from Peak IIIA are presented in Figure 8. The EI mass spectrum (Figure $8 a$ ) is characterized by the expected pentafluorobenzyl cation at $m / z 181$, which confirms that this compound is a PFBHA derivative of a carbonyl. However, the absence of ions at $\mathrm{m} / \mathrm{z} 73$ and 75 indicates that the compound is not the PFBHA-BSTFA derivative of a hydroxycarbonyl. The absence of an ion at $m / z 340$ further indicates that it is not the PFBHA-BSTFA derivative of 2-hydroxy-2-methylpropanal. The PFBOH CI ion trap mass spectrum is characterized by ions at $m / z 266$ and 464 (Figure 8b). The absence of $(\mathrm{M}-15)^{+}$and $(\mathrm{M}+181)^{+}$ions at $\mathrm{m} / \mathrm{z} 340$ and 536 provides further evidence that the mass spectrum is not of the PFBHA-BSTFA derivative of 2-hydroxy-2-methylpropanal. If the $\mathrm{m} / \mathrm{z} 464$ ion is the $(\mathrm{M}+181)^{+}$ion of a

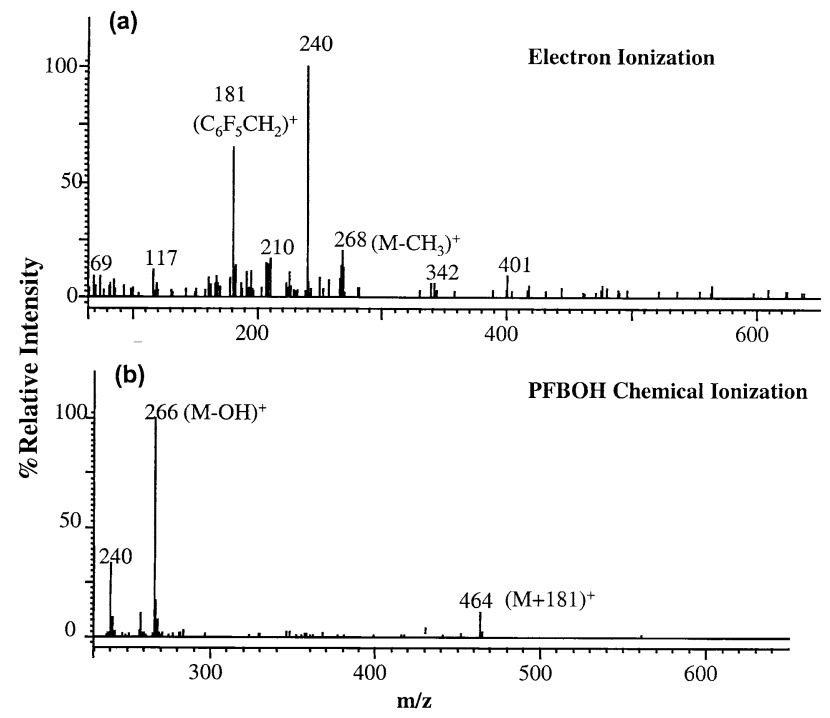

Figure 8. Electron ionization (a) and $\mathrm{PFBOH}$ chemical ionization (b) mass spectra of Peak IIIA 1 in Figure 7. 
Table 3. Comparison of signal:noise ratio for PFBHA- and PFBHA-BSTFA derivatives of hydroxycarbonyls and keto-acids

\begin{tabular}{|c|c|c|c|c|c|c|}
\hline \multirow[b]{3}{*}{ Analyte } & \multirow{3}{*}{$\begin{array}{c}\text { Concentration } \\
(\mathrm{pg} / \mu \mathrm{L})\end{array}$} & \multicolumn{5}{|c|}{ Signal:noise ratio } \\
\hline & & \multicolumn{2}{|c|}{ PFBHA-derivative } & \multicolumn{3}{|c|}{ PFBHA-BSTFA-derivative } \\
\hline & & $(\mathrm{M}+\mathrm{H})^{+}$ & $(\mathrm{M}-\mathrm{OH})^{+}$ & $(\mathrm{M}+\mathrm{H})^{+}$ & $\left(\mathrm{M}-\mathrm{CH}_{3}\right)^{+}$ & $(\mathrm{M}-89)^{+}$ \\
\hline Glycolaldehyde & 24 & 2,1 & 3,2 & 28,29 & 29,40 & 19,13 \\
\hline Hydroxyacetone & 24 & 3,3 & 6,2 & 23 & 13 & 13 \\
\hline 1-hydroxy-2-butanone & 24 & 1,1 & 2,1 & 1,8 & 1,10 & 1,8 \\
\hline 4-hydroxy-3-methyl-2-butanone & 26 & 2 & 1 & 8 & 7 & 7 \\
\hline 3-hydroxy-3-methyl-2-butanone & 23 & 1 & 21 & 1 & 1 & 23 \\
\hline Pyruvic acid & 50 & 1 & 1 & 71 & 12 & 18 \\
\hline
\end{tabular}

PFBHA-derivative, then the molecular weight of the derivative would be $283 \mathrm{Da}$, which is the molecular weight of the PFBHA derivative of 2-hydroxy-2-methylpropanal. Together, the data indicate that this compound is the PFBHA derivative of 2-hydroxy-2-methylpropanal. Thus, the ion at $\mathrm{m} / \mathrm{z} 266$ in both the methane and PFBOH CI mass spectra is likely an $(\mathrm{M}-\mathrm{OH})^{+}$ion. The $m / z 266$ ion is likely common to both the PFBHA derivative (IIIA $)_{1}$ ) and the PFBHA-BSTFA derivative (IIIA $_{2}$ ) of 2-hydroxy-2-methylpropanal because it is a stable tertiary carbocation, formed by loss of $\mathrm{OH}$ from the PFBHA derivative and by loss of $\mathrm{Si}\left(\mathrm{CH}_{3}\right)_{3} \mathrm{O}$ from the PFBHA-BSTFA derivative.

These data indicate incomplete BSTFA derivatization of 2-hydroxy-2-methylpropanal in certain sample extracts, and thus the observation of the PFBHA and the PFBHA-BSTFA derivatives. The fact that both peaks are observed, and that the peak shape for the PFBHAderivative is good, invokes the question "why derivatize with BSTFA?" In previous work, we demonstrated that derivatization with BSTFA improves the chromatography and sensitivity for a many hydroxycarbonyls and aldo- and keto-acids on a DB-5 ms column [18]. However, because the chromatography for PFBHA derivatives of hydroxycarbonyls and aldo- and keto-acids could be different on a DB-17 ms column, we compared the signal to noise ratio for PFBHA and PFBHA-BSTFA derivatives of hydroxycarbonyls and a keto-acid on a DB-17 ms column. These data are presented in Table 3 for the $(\mathrm{M}+\mathrm{H})^{+}$and $(\mathrm{M}-\mathrm{OH})^{+}$ions from the PFBHA derivatives and the $(\mathrm{M}+\mathrm{H})^{+},(\mathrm{M}-15)^{+}$, and $(\mathrm{M}-89)^{+}$ ions from the PFBHA-BSTFA derivatives. In cases in which the $E$ - and $Z$-isomers were resolved, two values are reported. The data demonstrate that BSTFA derivatization improves the signal to noise ratio for glycolaldehyde, hydroxyacetone, 1-hydroxy-2-butanone, 4-hydroxy-3methyl-2-butanone, and pyruvic acid, but the signal to noise ratio is the same with or without BSTFA derivatization for 3-hydroxy-3-methyl-2-butanone. The tertiary - $\mathrm{OH}$ group on 3-hydroxy-3-methyl-2-butanone is probably protected from interaction with the column, and thus the chromatography and sensitivity are good without BSTFA derivatization. In our work, BSTFA derivatization is required because glycolaldehyde, hydroxyacetone, and pyruvic acid are also photooxidation products of biogenic and anthropogenic hydrocarbons.

\section{Confirmation of the Identity of}

2-Hydroxy-2-Methylpropanal

In order to confirm the identification of the $\mathrm{C}_{4}$-hydroxycarbonyl that is produced from the reaction of $\mathrm{MBO}$ with $\cdot \mathrm{OH}$, we synthesized 2-hydroxy-2-methylpropanal. Chamber experiments and analysis of ambient air samples were concluded prior to the availability of an authentic standard 2-hydroxy-2-methylpropanal. We were unable to retroactively analyze the sample extracts because of concerns about possible degradation of the analyte in the extract. In Figure 9, we present the $\mathrm{EI}$ and methane CI ion trap mass spectra of the PFBHA derivative of 2-hydroxy-2-methylpropanal. The relative retention time of the gas chromatographic peak is the

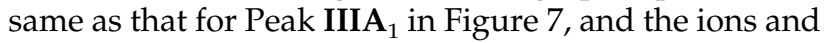
the relative intensity of the ions in the mass spectra are nearly identical to the spectra for Peak IIIA $_{1}$, as presented in Figures $7 \mathrm{~b}$ (methane CI) and 8a (EI). The EI mass spectra area characterized by the presence of ions at $\mathrm{m} / \mathrm{z} 268,240$ and 181 . The ions at $\mathrm{m} / \mathrm{z} 268$ and 240 are the $\left(\mathrm{M}-\mathrm{CH}_{3}\right)^{+}$and $\left[\mathrm{M}-\mathrm{CH}_{3}-(\mathrm{CO})_{2}\right]^{+}$ion of the PFBHA derivative of 2-hydroxy-2-methyl propanal, respectively. The only ion, and hence the base peak in the

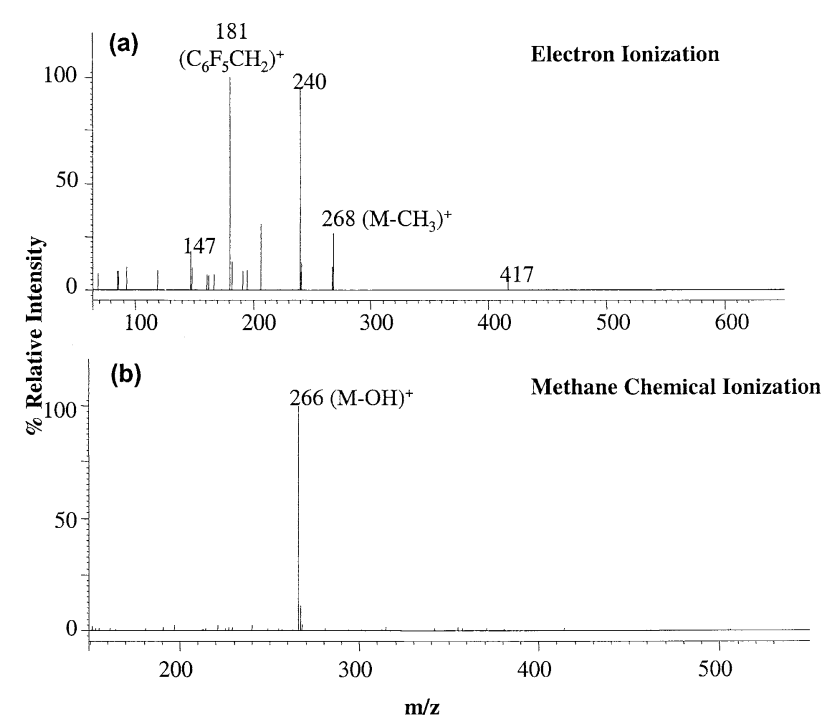

Figure 9. Electron ionization (a) and methane chemical ionization (b) mass spectra of the PFBHA derivative of 2-hydroxy-2methylpropanal. 


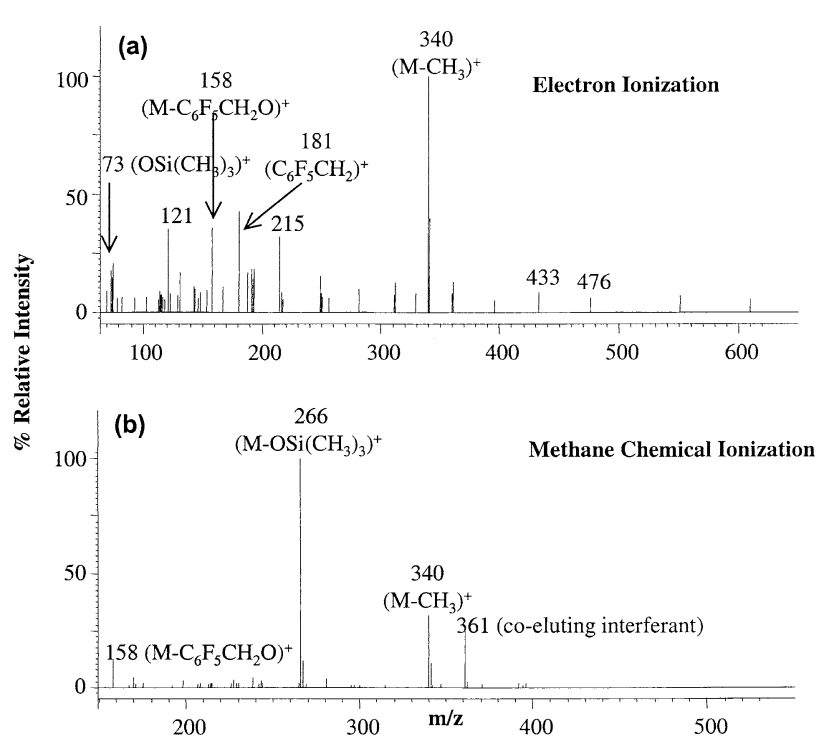

Figure 10. Electron ionization (a) and methane chemical ionization (b) mass spectra of the PFBHA-BSTFA derivative of 2-hydroxy-2-methylpropanal.

methane CI mass spectra is the ion at $m / z 266$ due to the loss of a hydroxyl group from the PFBHA derivative of 2-hydroxy-2-methylpropanal. In Figure 10, we present the EI and methane CI ion trap mass spectra for the PFBHA-BSTFA derivative of 2-hydroxy-2-methylpropanal. The relative retention time of the gas chromato-

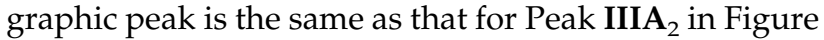
7 , and again the ions and the relative intensity of the ions in the mass spectra are nearly the same as the spectra for Peak IIIA, as shown in Figures 4a (methane $\mathrm{CI}$ ) and $6 \mathrm{~B}$ (EI). In addition to the presence of characteristic ions at $\mathrm{m} / \mathrm{z} 73$ and 181 ions, ions at $\mathrm{m} / \mathrm{z} 158$ $\left(\mathrm{M}-\mathrm{C}_{6} \mathrm{~F}_{5} \mathrm{CH}_{2} \mathrm{O}\right)^{+}$and $340\left(\mathrm{M}-\mathrm{CH}_{3}\right)^{+}$are observed. The methane $\mathrm{CI}$ mass spectra exhibit ions at $\mathrm{m} / \mathrm{z} 340$ and 266. In the mass spectra of the authentic standard, we observe ions at $m / z 158$ and 361 which are not present in the methane CI mass spectra of the sample extract. The ion at $m / z 158$ is presumably due to the loss of $\mathrm{C}_{6} \mathrm{~F}_{5} \mathrm{CH}_{2} \mathrm{O}$, and the ion at $\mathrm{m} / z 361$ is due to a co-eluting interferant. Thus, the presence of ions at similar ion intensities in PFBHA and PFBHA-BSTFA derivative in the EI and methane CI mass spectra of the authentic standard, compared to derivatives in the sample extract, confirm the presence of 2-hydroxy-2-methylpropanal.

\section{Semi-Quantification of 2-Hydroxy-2- Methylpropanal in Air Samples Collected at the Blodgett Forest}

Since an authentic standard of 2-hydroxy-2-methylpropanal was not available at the time that Blodgett Forest samples were quantified, we evaluated the use of 3hydroxy-3-methyl-2-butanone and 4-hydroxy-4-methyl-2-pentanone, two ketones with tertiary $-\mathrm{OH}$ groups as surrogates. These compounds were chosen because we were unable to locate a commercial source of an aldehyde with a tertiary $-\mathrm{OH}$ group, which would be more structurally similar to 2-hydroxy-2-methylpropanal than a ketone. We evaluated whether a ketone is a reasonable surrogate for a structurally similar aldehyde by comparing the relative response factors between methacrolein and methyl vinyl ketone. Since the total concentration of the analyte derivative will be the concentration of the PFBHA derivative and the concentration of the PFBHA/BSTFA due to the inconsistent BSTFA derivation, and the response factor of a ketone with a tertiary $-\mathrm{OH}$ group to the internal standard may be compound dependent, we also compared the difference between the relative response factors of the two ketones with tertiary $-\mathrm{OH}$ by using a predominant ion in the mass spectra of the PFBHA derivative, or the PFBHA/BSTFA derivative as a single quantifying ion, or by using a sum of the ions. We obtained a $6 \%$ difference and a $9 \%$ relative standard deviation between relative response factors obtained by analyzing standards of methacrolein and methyl vinyl ketone ranging from 241 to $1024 \mathrm{pg} / \mu \mathrm{L}$. The small difference between the relative response factors of the two compounds suggests similar responses between a structurally similar aldehyde and a ketone. We compared the relative response factor obtained by using the $(\mathrm{M}-17)^{+}$ ion of the PFBHA derivative, and the $(\mathrm{M}-15)^{+}$or $(\mathrm{M}-89)^{+}$ions of the PFBHA/BSTFA derivatives of 3-hydroxy-3-methyl-2-butanone and 4-hydroxy-4-methyl2-pentanone, each separately as the quantifying ions, and as the sum of these ions. For concentrations ranging from 115 to $864 \mathrm{pg} / \mu \mathrm{L}$, the \% difference between the relative response factor for the $(\mathrm{M}-17)^{+},(\mathrm{M}-15)^{+}$, and the $(\mathrm{M}-89)^{+}$ions differed by 49,125 , and $120 \%$, respectively. This difference between the compounds was reduced to $16 \%$ by using the sum of the three ions. We thus chose to quantify by using the sum of the ions to reduce differences that may exist among structurally similar molecules, and to account for inconsistent BSTFA derivatization. We constructed standard curves using the sum of the areas of the $(\mathrm{M}-15)^{+}$and $(\mathrm{M}-89)^{+}$ ions from PFBHA-BSTFA derivative and the $(\mathrm{M}-\mathrm{OH})^{+}$ ion from the PFBHA derivative of 3-hydroxy-3-methyl-2butanone. The response factor for the sum of the equivalent ions from the PFBHA and PFBHA-BSTFA derivatives of 2-hydroxy-2-methylpropanal was substituted into the regression equation to semi-quantify 2-hydroxy-2-methylpropanal.

We provide evidence that the method of semi-quantification is reasonable by comparing the values obtained by our method to values obtained by using FT-IR for the concentration of 2-hydroxy-2-methylpropanal produced from the reaction of $\cdot \mathrm{OH}$ with $\mathrm{MBO}$ in an environmental chamber. The concentration obtained by using FT-IR was estimated from calibration data obtained in a previous experiment, and a yield of $29 \pm 3 \%$. By using the PFBHA-BSTFA method we measured mean $n=2$ ) concentrations of 266,396 , and $483 \mathrm{ppbv}$ for three samples, compared to $380 \mathrm{ppv}, 570 \mathrm{ppv}$, and $820 \mathrm{ppbv}$ by using the FT-IR method. Thus, assuming a yield of 


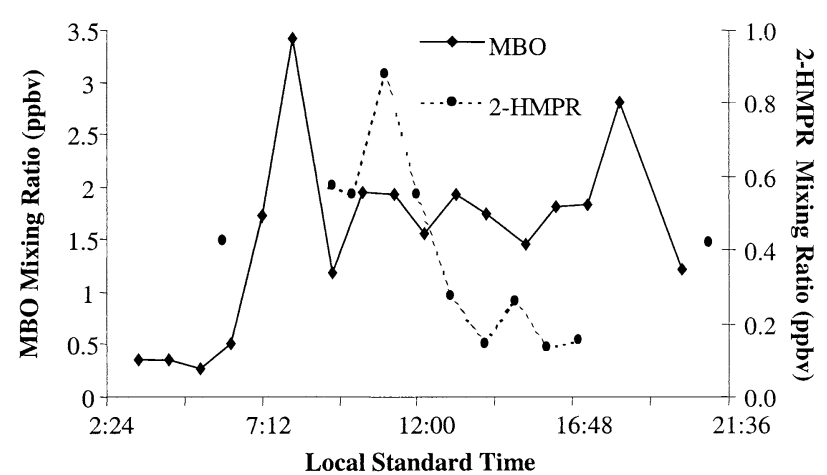

Figure 11. Mixing ratios of 2-methyl-3-buten-2-ol (MBO) and its photooxidation product, 2-hydroxy-2-methylpropanal (2-HMPR), in Blodgett Forest air.

$29 \%$, the semi-quantification method is 30 to $41 \%$ lower than the FT-IR method. However, Alvarado et al. [14], using silylation and GC-FID, estimated that the 2-hydroxy-2-methylpropanal yield was only $19 \pm 9 \%$. The values reported by the FT-IR method would then be lower $(249,373$, and $537 \mathrm{ppbv}$ in the three sample extracts). In this case, the \% relative differences would be $6-10 \%$ between the two methods. The concentrations of 2-hydroxy-2-methylpropanal semi-quantified by the PFBHA-BSTFA, compared to the FT-IR method thus varies from 6 to $41 \%$, depending on the assumed yield of hydroxy-2-methyopropanal.

We present the mixing ratios of MBO (Gunnar Schade, personal communication) and 2-hydroxy-2-methylpropanal (2-HMPR) as a function of time in Figure 11. Overall, the average ratio of [2-hydroxy-2-methylpropanal]/[MBO] was $0.33 \pm 0.26$, providing further support that the semi-quantification of 2-hydroxy-2-methylpropanal is reasonable, since the expected yield of 2-hydroxy-2-methylpropanal from the reaction of $\mathrm{MBO}$ with $\cdot \mathrm{OH}$ is $0.19-0.35[11,13,14]$.

The mixing ratios of 2-hydroxy-2-methylpropanal increase or decrease approximately 2 to $2.5 \mathrm{~h}$ after increases and decreases in $\mathrm{MBO}$, which is consistent with a local source of $\mathrm{MBO}$ and in situ photochemical production of 2-hydroxy-2-methylpropanal. Additionally, the ratio of [2-hydroxy-2-methylpropanal]/[MBO] was higher (0.5-0.9) during early morning and nighttime compared to mid-day and early evening $(\leq 0.2)$. This is expected, because during daytime MBO is continuously emitted by trees [10], 2-hydroxy-2-methylpropanal is produced from the reaction of $\mathrm{MBO}$ with $\cdot \mathrm{OH}$, and the build-up of both is limited by reactions with $\cdot \mathrm{OH}[11$, $13,14]$. However, after sunset, emissions of $\mathrm{MBO}$ cease, MBO is depleted by reactions with $\mathrm{O}_{3}$ and $\mathrm{NO}_{3}$, and small amounts of 2-hydroxy-2-methylpropanal may be produced by reaction of $\mathrm{MBO}$ with $\mathrm{O}_{3}[12-14,33,34]$. Additionally, changing wind direction at night could bring in air with higher 2-hydroxy-2-methylpropanal mixing ratios from surrounding forests [2]. Therefore, at night $\mathrm{MBO}$ will be depleted more rapidly than 2-hydroxy-2-methylpropanal, and small amounts of 2-hydroxy-2-methylpropanal may be formed or carried in by wind, creating higher [2-hydroxy-2-methylpropanal]/ [MBO] ratios. When the sun rises, $\mathrm{MBO}$ and $\cdot \mathrm{OH}$ mixing ratios will increase, 2-hydroxy-2-methylpropanal will still be formed by $\mathrm{MBO}$ reactions, but will also be depleted by reaction with $\cdot \mathrm{OH}$ and possibly by photolysis, and by afternoon the [2-hydroxy-2-methylpropanal]/ [MBO] ratio will be significantly smaller.

\section{Conclusions}

Interpretation of EI, methane $\mathrm{CI}$, and $\mathrm{PFBOH} \mathrm{CI}$ ion trap mass spectra of PFBHA and PFBHA-BSTFA derivatives was critical for: (1) Identifying the structure of the $\mathrm{C}_{4}$-hydroxycarbonyl produced by the reaction of $\cdot \mathrm{OH}$ with $\mathrm{MBO}$; (2) solving co-elution problems on a DBXLB GC column; and (3) developing a method to semiquantify 2-hydroxy-2-methylpropanal in air. Ion-molecule reactions which occur only in an ion trap mass spectrometer were facilitated by bleeding $\mathrm{PFBOH}$ directly in to the ion trap. The resulting $(\mathrm{M}+181)^{+}$ions aided in determining the structure of the $\mathrm{C}_{4}$-hydroxycarbonyl and were essential for differentiating the PFBHA derivative of 2-hydroxy-2-methylpropanal from the PFBHABSTFA derivative in the absence of an authentic standard. Semi-quantification of 2-hydroxy-2-methylpropanal by FT-IR and by the method developed here compared favorably. We thus used the method to obtain the first diurnal measurements of 2-hydroxy-2-methylpropanal in ambient air, and to gain insight into the relationship between 2-hydroxy-2-methylpropanal and its precursor, $\mathrm{MBO}$, in the ambient atmospheric environment.

\section{Acknowledgments}

This work was supported by the NSF Atmospheric Chemistry Program (grant no. 0003137) and an NIEHS Training Grant (grant no. T32 ES07059). The authors sincerely thank Dr. Michael H. Nantz for overseeing the synthesis of 2-hydroxy-2-methylpropanal, Dr. Allen Goldstein and Dr. Gunnar Schade for MBO data from the Blodgett Forest and for useful discussions, and Dr. Roger Atkinson for assistance with chamber experiments and review of the manuscript. They also thank Elsie Ovrahim for assistance with sample collection.

\section{References}

1. Saxena, P.; Hildemann, L. M. J. Atmos. Chem. 1996, 24, 57-109.

2. Lamanna, M. S.; Goldstein, A. H. J. Geophys. Res. 1999, 104, 21247-21262.

3. Jenkin, M. E.; Clemitshaw, K. C. Atmos. Environ. 2000, 34, 2499-2527.

4. Trainer, M.; Williams, E. J.; Parrish, D. D.; Buhr, M. P.; Allwine, E. J.; Westberg, H. H.; Fehsenfeld, F. C.; Liu, S. C. Lett. Nature 1987, 329, 705-707.

5. Chameides, W. L.; Lindsay, R. W.; Richardson, J.; Kiang, C. S. Science 1988, 241, 1473-1475.

6. Andreae, M. O.; Crutzen, P. J. Science 1997, 276, 1052-1058.

7. Pierce, T.; Geron, C.; Bender, L.; Dennis, R.; Tonnesen, G.; Guenther, A. J. Geophys. Res. 1998, 103, 25611-25629.

8. Atkinson, R. J. Phys. Chem. Ref. Data 1997, 26, 215-290.

9. Goldan, P. D.; Kuster, W. C.; Fehsenfeld, F. C. Geophys. Res. Lett. 1993, 20, 1039-1042. 
10. Harley, P.; Fridd-Stroud, V.; Greenberg, J.; Guenther, A.; Vasconcellos, P. J. Geophys. Res. 1998, 103, 25479-25486.

11. Ferronato, C.; Orlando, J. J.; Tyndall, G. S. J. Geophys. Res. 1998, 103, 25579-25586.

12. Fantechi, G.; Jensen, N. R.; Hjorth, J.; Peeters, J. Int. J. Chem. Kinet. 1998, 30, 589-594.

13. Fantechi, G.; Jensen, N. R.; Hjorth, J.; Peeters, J. Atmos. Environ. 1998, 32, 3547-3556.

14. Alvarado, A.; Tuazon, E. C.; Aschmann, S. M.; Arey, J.; Atkinson, R. Atmos. Environ. 1999, 33, 2893-2905.

15. Spaulding, R. S.; Talbot, R.; Charles, M. J. Environ. Sci. Technol., unpublished.

16. Yu, J.; Jeffries, H. E.; Sexton, K. G. Atmos. Environ. 1997, 31, 2261-2280.

17. Yu, J.; Flagan, R. C.; Seinfeld, J. H. Environ. Sci. Tech. 1998, 32, 2357-2370.

18. Spaulding, R. S.; Frazey, P. A.; Rao, X.; Charles, M. J. Anal. Chem. 1999, 71, 3420-3427.

19. Frazey, P.; Rao, X.; Spaulding, R.; Beld, B.; Charles, M. J. Int. J. Mass Spectrom. 1999, 191, 343-357.

20. Rao, X.; Kobayashi, R.; White, R.; Spaulding, R.; Frazey, P.; Charles, M. J. J. AOAC Int. 2001, 84, 699-705.

21. Cancilla, D. A.; Chou, C.-C.; Barthel, R.; Que Hee, S. S. J. AOAC Int. 1992, 75, 842-854.
22. Willard, H. H.; Merritt, L. L., Jr.; Dean, J. A.; Settle, J. Instrumental Methods of Analysis. Wadsworth, Inc: Belmont, 1988.

23. Atkinson, R.; Carter, W. P. L.; Winer, A. M.; Pitts, J. N., Jr. APCA Notebook 1981, 31, 1090-1092.

24. Schade, G. W.; Goldstein, A. H.; Gray, D. W.; Lerdau, M. T. Atmos. Environ. 2000, 34, 3535-3544.

25. Cofer, W. R. I.; Collins, V. G.; Talbot, R. W. Environ. Sci. Technol. 1985, 19, 557-560.

26. Klemm, O.; Talbot, R. W. J. Atmos. Chem. 1991, 13, 325-342.

27. Baeckstrom, P.; Li, L. Tetrahedron 1991, 47, 6533.

28. Fessner, W. D.; Gosse, C.; Jaeschke, G.; Eyrisch, O. Eur. J. Org. Chem. 2000, 1, 125-132.

29. Feedman, H. H.; Dubois, R. A. Tetrahedron Lett. 1976, 17, 3535.

30. Yu, J.; Jeffries, H. E.; LeLacheur, R. M. Environ. Sci. Technol. 1995, 29, 1923-1932.

31. LeLacheur, R. M.; Sonnenberg, L. B.; Singer, P. C.; Christman, R. F.; Charles, M. J. Environ. Sci. Technol. 1993, 27, 2745-2753.

32. Chien, C.-J.; Charles, M. J.; Sexton, K. G.; Jeffries, H. E. Environ. Sci. Technol. 1998, 32, 299-309.

33. Grosjean, E.; Grosjean, D. Int. J. Chem. Kinet. 1994, 26, 11851191.

34. Grosjean, E.; Grosjean, D. J. Geophys. Res. 1995, 100, 2281522820 